
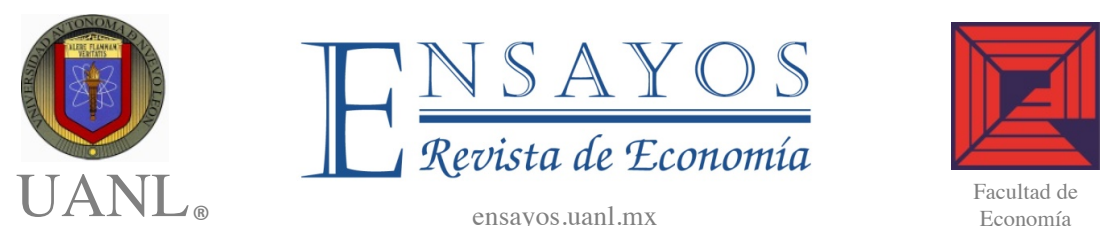

\title{
Percepción de inseguridad versus tasa delictiva; ¿qué afecta más la economía mexicana?
}

\section{Insecurity perception versus crime rate; ¿which has a bigger impact on Mexico's economy?}

Pablo Quezada*† Miguel Santillan* Ricardo Hinojosa* Jorge Rada*

\begin{tabular}{|c|c|}
\hline $\begin{array}{l}\text { Información del } \\
\text { artículo }\end{array}$ & Resumen \\
\hline Recibido: & El propósito de este estudio es comparar el impacto de \\
\hline 27 agosto 2018 & $\begin{array}{l}\text { la percepción de la inseguridad contra la tasa delictiva } \\
\text { en indicadores económicos, que reflejan los medios por }\end{array}$ \\
\hline Aceptado: & los cuales estas dos variables podrían afectar la \\
\hline 24 abril 2019 & $\begin{array}{l}\text { economía. A través de un análisis de datos panel, } \\
\text { considerando los } 32 \text { estados de la república mexicana }\end{array}$ \\
\hline $\begin{array}{l}\text { Clasificación JEL: } \\
\mathrm{H} 10 ; \mathrm{O} 20\end{array}$ & $\begin{array}{l}\text { en el periodo } 2011 \text { - 2016, se encuentra que la } \\
\text { percepción de la inseguridad afecta más a la economía } \\
\text { que la tasa delictiva. }\end{array}$ \\
\hline
\end{tabular}

Palabras clave:

Percepción de la inseguridad; Tasa delictiva; Heurísticas

\footnotetext{
* Instituto Tecnológico y de Estudios Superiores de Monterrey (ITESM). Campus Monterrey.

${ }^{\dagger}$ Email : pablo.quezada@itesm.mx
} 


\begin{tabular}{|c|c|}
\hline Article information & Abstract \\
\hline $\begin{array}{l}\text { Received: } \\
27 \text { august } 2018\end{array}$ & $\begin{array}{l}\text { The purpose of this study is to compare the impact of } \\
\text { insecurity perception against the crime rate in }\end{array}$ \\
\hline $\begin{array}{l}\text { Accepted: } \\
24 \text { april } 2019\end{array}$ & economic indicators that reflect the channels in which \\
\hline $\begin{array}{l}\text { JEL Classification: } \\
\mathrm{H} 10 ; \mathrm{O} 20\end{array}$ & $\begin{array}{l}\text { Mexican republic in the period } 2011-2016 \text {, it is found } \\
\text { that the perception of insecurity has a higher impact on } \\
\text { the economy than the crime rate. }\end{array}$ \\
\hline $\begin{array}{l}\text { Keywords: } \\
\text { Insecurity perception; } \\
\text { Crime rate; Heuristics }\end{array}$ & \\
\hline
\end{tabular}

\section{Introducción}

La inseguridad es uno de los temas que más preocupa a los mexicanos. El 32\% de los mexicanos considera que la delincuencia e inseguridad son el mayor problema en México, por encima de la corrupción (16\%) y bajos salarios (12\%); además, la mayoría piensa que la inseguridad va en aumento (IPSOS, junio 2017).

Dado que la inseguridad afecta la economía a través del impacto que tiene en las decisiones de las personas que la integran y los hallazgos de la economía conductual indican que, en muchos casos, éstas usan heurísticas basadas en percepciones más que en hechos para tomar decisiones, se sospecha que la percepción de la inseguridad podría afectar la economía más que la tasa delictiva.

El propósito de este estudio es comparar el impacto de la percepción de la inseguridad contra la tasa delictiva en indicadores económicos, que reflejan los medios por los cuales estas dos variables podrían afectar la economía mexicana.

Se encuentra que la percepción de la inseguridad afecta más la economía mexicana que las tasas delictivas consideradas en este estudio. Las actividades económicas más afectadas por la percepción de la inseguridad resultan ser la cantidad de empresas registradas en el Sistema de Información Empresarial Mexicano (SIEM) y el producto interno bruto de servicios de alojamiento temporal y de preparación de alimentos y bebidas en millones de MXN.

Esto implica que, si los gobiernos en México quieren ver sus esfuerzos para combatir la inseguridad reflejados en la economía mexicana, tendrán que considerar reducir la percepción de la inseguridad, sin olvidar reducir las tasas delictivas y poner más énfasis en lo primero que en lo segundo. 
El resto del artículo está estructurado de la siguiente manera: en la primer sección se explica cómo se mide la percepción de la inseguridad en México; en la segunda, se discute si existe una relación entre la percepción de la inseguridad y la tasa delictiva en México; en la tercera, se menciona cómo es que la percepción de la inseguridad y la tasa delictiva podrían afectar la economía mexicana; en la cuarta, se detalla el análisis empírico realizado para probar la hipótesis de este estudio; en la quinta se muestra los resultados del análisis empírico; en la sexta se incluye un análisis de sensibilidad del estudio empírico.

\section{I. ¿Cómo se mide la percepción de la inseguridad en México?}

En México, desde 2011 se realiza anualmente la Encuesta Nacional de Victimización y Percepción sobre la Seguridad Pública (ENVIPE), para monitorear y tratar de entender este fenómeno.

Para medir la percepción de la inseguridad, los encargados de realizar esta encuesta le preguntan a los encuestados si consideran que vivir en cierta zona geográfica es inseguro. Es decir, si contestan con la opción (2) a la pregunta 4.3 de la ENVIPE de 2016, 2015, 2014, y 2013, que dice: "En términos de delincuencia, considera que vivir en (ámbito geográfico) es: (1) ¿seguro? (2) ¿inseguro? (3) No sabe/No responde" y si contestan con la opción (2) a la pregunta 4.4 de la ENVIPE 2012 y la pregunta 4.3 de la ENVIPE 2011: “¿Considera que vivir en (ámbito geográfico) es: (1) seguro? (2) inseguro? (3) No sabe/No responde"

Cabe notar que en las ENVIPE correspondientes a los años 2016, 2015, 2014 y 2013 se aclara que la pregunta 4.3, que se menciona en el párrafo previo, es "En términos de delincuencia" a diferencia de la pregunta 4.4 de la ENVIPE 2012 y la pregunta 4.3 de la ENVIPE 2011, donde no se realiza esta a aclaración. Debido a que es poco probable que el encuestado no relacione la pregunta con la delincuencia, porque sabe de antemano que la encuesta es sobre la victimización y esta generalmente se entiende en términos de delincuencia, se asume que las preguntas son equivalentes, independientemente si se hizo o no la aclaración.

Bajo estas consideraciones, más de la mitad de los mexicanos considera que vivir en su país es inseguro desde 2011 hasta el 2016, y en el período de 2013 a 2016, la fracción aumenta a más de un tercio, tal y como se muestra en el gráfico 1: 


\section{Gráfico 1}

Fracción de la población de los Estados Unidos Mexicanos que considera inseguro vivir en los Estados Unidos Mexicanos

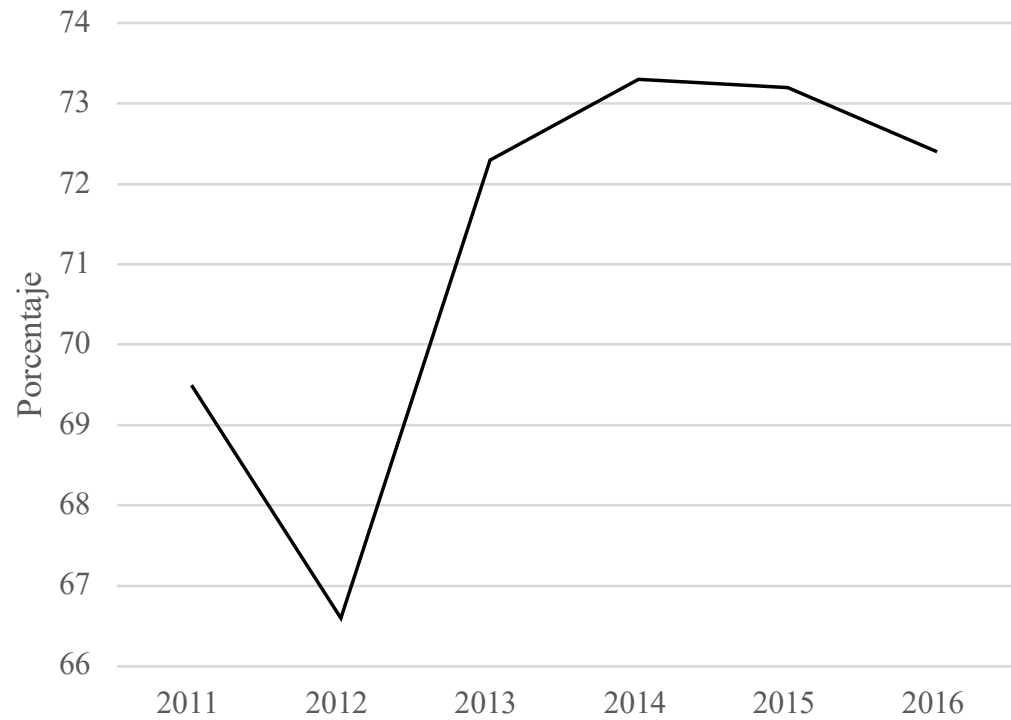

Fuente: elaboración propia con datos calculados por el Instituto Nacional de Estadística y Geografía (INEGI) considerando los resultados de La Encuesta Nacional de Victimización y Percepción sobre Seguridad Pública (ENVIPE) de los años 2011-2016.

\section{II. ¿Cuál es la relación entre la percepción de la inseguridad y la tasa delictiva?}

De 2011 a 2016, la tasa de incidencia delictiva por cada 100 mil habitantes calculada por el INEGI con datos de la ENVIPE se ha mantenido en el rango de entre 29,200 y 41,654, tal y como se puede observar en el gráfico 2.

Desde una perspectiva económica tradicional, la percepción de la inseguridad debería estar relacionada con la tasa delictiva. Si las personas son racionales en el sentido económico, es decir, que lo saben todo, toman decisiones a través de un procedimiento lógico y no se equivocan, su percepción de inseguridad debería estar en función de la tasa delictiva y la relación esperada entre estas dos variables debería ser directa. Entre mayor sea la tasa delictiva, mayor debería ser la percepción de la inseguridad. Si las personas realmente consideran las tasas delictivas calculadas por el INEGI con datos de la ENVIPE, para evaluar la seguridad de una zona geográfica en determinado momento, la relación entre la percepción de la inseguridad y la tasa delictiva debería ser directa y con rezago de un año, puesto que en el momento de la entrevista la persona solo puede saber la tasa delictiva del año anterior. Por 
supuesto que una persona racional en el sentido económico, no solo tomará en cuenta la tendencia histórica de las tasas delictivas calculadas por el INEGI con datos de la ENVIPE. Adicional a esto, deberá considerar las políticas públicas que se han llevado a cabo en el período, desde que salieron los últimos resultados de la ENVIPE y se publicaron las tasas delictivas, hasta el momento de la entrevista. Sin embargo, aún así, se esperaría una relación directa entre la percepción de la inseguridad y las tasas delictivas, porque ninguna política pública puede resolver el problema de la inseguridad en menos de un año, y una persona racional, en el sentido económico, lo debe saber.

\section{Gráfico 2}

Tasa de Incidencia Delictiva por cada 100 mil habitantes en los Estados Unidos Mexicanos

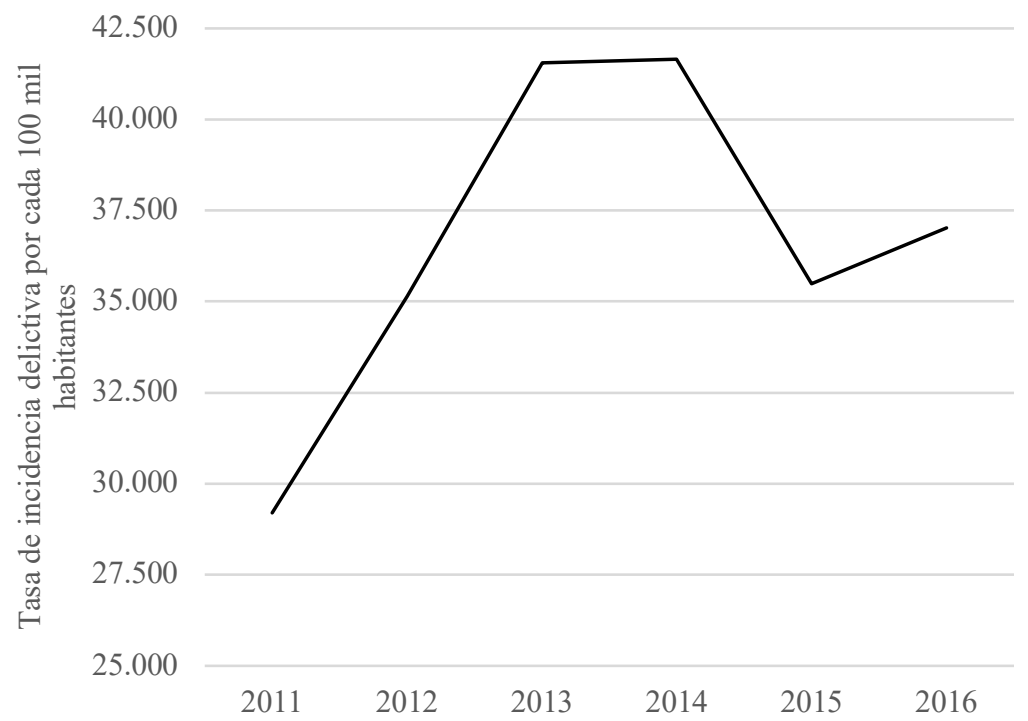

Fuente: elaboración propia con datos calculados por el Instituto Nacional de Estadística y Geografía (INEGI) considerando los resultados de La Encuesta Nacional de Victimización y Percepción sobre Seguridad Pública (ENVIPE) de los años 2011-2016.

El procedimiento descrito en el párrafo anterior no es más que una aplicación de la teoría de probabilidad de Bayes, para calcular la probabilidad de ser víctima de un acto delictivo en cierta zona geográfica y en un determinado momento. De acuerdo con esta teoría, las personas deberían considerar las probabilidades a priori calculadas con datos históricos (en este caso, los resultados de la encuesta ENVIPE) y las probabilidades posteriores calculadas con nueva información (en este caso, las políticas públicas que se están llevando a cabo en el presente, para reducir la inseguridad). 
En consecuencia, ¿qué se observa en la realidad? El gráfico 3 muestra la tasa de incidencia delictiva por cada 100 mil habitantes calculada por la INEGI con datos de la ENVIPE, para los Estados Unidos Mexicanos, contrastada con la fracción de la población que considera inseguro vivir en este país.

Tal y como se puede observar, pareciera ser que la relación entre la percepción de la inseguridad y la tasa delictiva es directa y que tiene un año de rezago; sin embargo, es probable que la relación no sea lineal, puesto que los cambios en la tasa delictiva no conllevan siempre a cambios en la percepción de la inseguridad en la misma proporción. La tasa delictiva aumentó $20 \%$ de 2011 a 2012; mientras que la percepción de la inseguridad, solo aumentó $8.5 \%$ de 2012 a 2013. Posteriormente, la tasa delictiva aumentó 18\% de 2012 a 2013, mientras que la percepción de la inseguridad solo aumentó $1 \%$. Además, este gráfico compara solamente dos series de tiempo de variables aleatorias muy cortas. La serie de tiempo a penas cuenta con 6 observaciones.

Para ver este grado de relación con más detalle, se calcula el coeficiente de correlación distancia desarrollado por Szekely et al. (2007), de la matriz de las percepciones de inseguridad y la matriz de las tasas de incidencia delictiva por cada 100 mil habitantes, de los 32 estados de la república mexicana para el período 2011-2016. El coeficiente de correlación distancia, a diferencia del coeficiente de correlación de Pearson, no asume que la relación entre las matrices de las variables aleatorias sea lineal. El coeficiente de correlación distancia está acotado entre 0 y 1 , donde un número cercano a 0 significa que las matrices de variables aleatorias que se están comparando tienen poca relación, y un número cercano a 1 significa que tienen mucha relación. Un coeficiente de correlación distancia igual a 0 significa que las matrices de variables aleatorias son independientes, en el sentido estadístico. El cálculo del coeficiente de correlación distancia para estas matrices resultó en 0.2088 . Esto significa que, considerando estas muestras, la percepción de la inseguridad y la tasa de incidencia delictiva por cada 100 mil habitantes no están muy relacionadas.

Esto concuerda con los hallazgos de Curiel y Bishop (2016). Estos autores muestran en su estudio para el caso de México que no existe una relación clara entre la percepción de la inseguridad tal y como se define en este artículo y las tasas delictiva. En este estudio, los autores comparan la ordenación de mayor a menor percepción de la inseguridad, contra la ordenación de mayor a menor tasa de incidencia delictiva de varios municipios en México, y encuentran que la relación de estas ordenaciones se asemeja a una relación aleatoria. Es decir, que el municipio con mayor percepción de inseguridad, puede estar relacionado con cualquier lugar de los municipios ordenados de mayor a menor tasa de incidencia delictiva. 


\section{Gráfico 3}

Tasa de incidencia delictiva por cada 100 mil habitantes y fracción de la población que considera inseguro vivir en su estado en los Estados Unidos Mexicanos

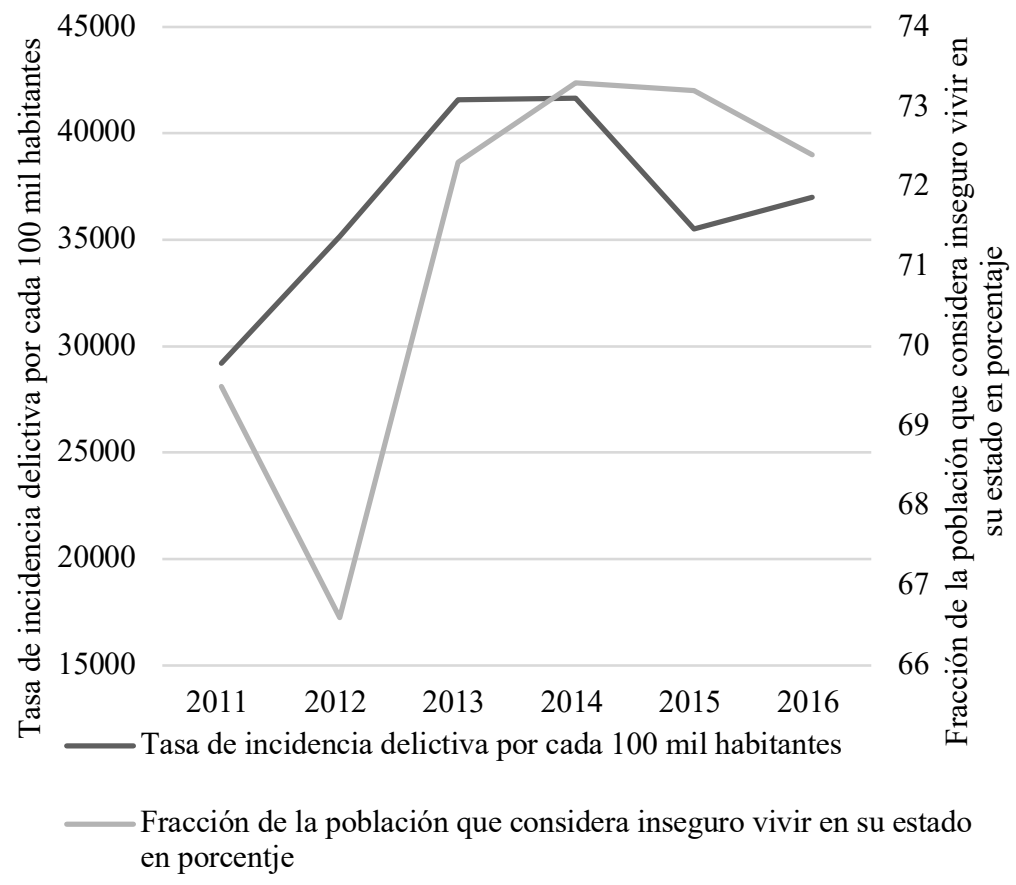

Fuente: elaboración propia con datos calculados por el Instituto Nacional de Estadística y Geografía (INEGI), considerando los resultados de La Encuesta Nacional de Victimización y Percepción sobre Seguridad Pública (ENVIPE) entre los años 2011-2016

Estos resultados indican que las personas no se están comportando como la teoría económica tradicional predice. Si así fuera, el coeficiente de correlación distancia de la matriz de las percepciones de inseguridad y la matriz de las tasas de incidencia delictiva por cada 100 mil habitantes de los 32 estados de la república mexicana, para el período 2011-2016, sería mayor, y Curiel y Bishop (2016) hubieran encontrado lo contrario a lo que hallaron en su estudio. ¿Qué explicación podría tener esta observación?

Es posible que la percepción de la inseguridad no esté relacionada o está débilmente relacionada con las tasas delictivas porque, en la mayoría de los casos, las personas utilizan heurísticas para evaluar la seguridad de una zona geográfica y posteriormente tomar decisiones económicas. Si este argumento es válido, la percepción de la inseguridad, tal y como se define en este estudio, debería afectar más la actividad económica que las tasas delictivas. 
Hay dos heurísticas que se podrían utilizar para evaluar la seguridad de cierta zona geográfica en determinado momento $\mathrm{y}$, posteriormente, tomar decisiones económicas: la heurística de la disponibilidad y la heurística de la confirmación.

La heurística de la disponibilidad de acuerdo con Tversky y Kahneman (1973) es una estrategia utilizada por las personas para contestar preguntas difíciles de forma rápida y satisfactoria. La estrategia consiste en sustituir una difícil pregunta, como: “¿Es segura tal zona geográfica?” por una más sencilla, como: “¿Qué dicen las noticias al respecto?”. Posteriormente, los recuerdos más recientes y salientes de la persona la ayudarán a contestar la pregunta sencilla. En este caso, si la persona leyó recientemente una noticia de un asesinato en cierta zona geográfica que le causó conmoción, es probable que cuando se le pregunte si dicha zona geográfica es segura, conteste que no.

Al igual que la heurística de la disponibilidad, la heurística de la confirmación de acuerdo con Bazerman y Moore (2008) es una estrategia utilizada por las personas para contestar preguntas difíciles de forma rápida y satisfactoria solo que, a diferencia de la primera, la segunda se basa en una creencia personal en lugar de recuerdos recientes y salientes. La estrategia consiste en sustituir una difícil pregunta, como: “¿Cuál es la probabilidad de que me asalten en un restaurant de cierta zona geográfica?” por una más sencilla, como: “ ¿Tal zona geográfica es segura?". Solo que, en este caso, para contestar la pregunta sencilla la persona buscará una creencia personal que a su vez será respaldada por recuerdos selectivos. Si la persona cree que tal zona geográfica es insegura, su mente realizará una búsqueda selectiva de evidencia que respalde dicha creencia (e. g., a un conocido lo asaltaron en un restaurant de dicha zona geográfica en algún momento, hay evidencia de actos vandálicos en dicha zona geográfica, etc.), y concluirá que dicha zona geográfica es insegura. Esta conclusión provocará que la persona no vaya al restaurant y decida hacer otra cosa con su tiempo.

Existe una amplia literatura que documenta, cómo el uso de heurísticas en ciertas circunstancias, resulta en juicios sesgados que provocan comportamientos contrarios a lo que predice la teoría económica tradicional. Para una extensa revisión literaria al respecto, véase Gilovich et al. (2002). La premisa de esta posible explicación está fundada en este conjunto de evidencia empírica.

Por estas razones, se plantea la hipótesis de que la percepción de la inseguridad afecta más a la economía que la tasa delictiva. 
Nótese, además, que la percepción de la inseguridad en este estudio, es una evaluación de la seguridad de una zona geográfica. Esto es diferente al miedo a ser víctima de un delito o el cálculo de la probabilidad de ser víctima de algún crimen, sobre lo cual existe suficiente literatura (véase Hale, 1996).

Este estudio busca complementar los hallazgos de Curiel y Bishop (2016), al extender el análisis a las implicaciones económicas que la poca relación existente entre la percepción de la inseguridad y las tasas delictivas, pueda tener.

\section{III. ¿Cómo es que la percepción de la inseguridad y la tasa delictiva podrían afectar la economía?}

Se asume que la percepción de la inseguridad y la tasa delictiva afectan cuatro actividades económicas, a través del mismo mecanismo que Ayala y Chapa (2012) proponen, para explicar cómo la probabilidad subjetiva de ser víctima de un delito afecta la demanda de entretenimiento. Para relacionar la hipótesis planteada en la introducción, con este mecanismo, se asume que la evaluación de la seguridad de una zona geográfica y/o la tasa delictiva de dicha zona geográfica, afecta directamente el cálculo de la probabilidad subjetiva de ser víctima de un delito. Es decir, si el individuo considera que vivir en cierta zona geografía es inseguro y/o la tasa delictiva en dicha zona geográfica es alta, es más probable que le asigne una probabilidad alta a la ocurrencia de ser víctima de un delito en el acto necesario para consumir el bien en cuestión, en dicha zona geográfica.

Para explicar cómo la probabilidad subjetiva de ser víctima de un delito afecta la demanda de entretenimiento, Ayala y Chapa (2012) proponen un modelo de demanda basado en la teoría económica tradicional; es decir, en la teoría de la utilidad esperada, donde el consumidor se encarga de maximizar una función de satisfacción sujeto a una restricción presupuestaria. La ventaja de este paradigma es que se puede adaptar a la demanda de otros bienes distintos al entretenimiento, sin cambiar sus propiedades ni las conclusiones.

Bajo este esquema, los individuos tendrían que maximizar la siguiente función de utilidad de elasticidades contantes:

$$
U(n, y)=\frac{\theta_{i} n^{\delta}}{\delta}+\frac{y^{\delta}}{\delta}
$$

Donde " $\mathrm{n}$ " es el bien de consumo de interés, " $\mathrm{y}$ " son todos los demás bienes de consumo que no sean "n", 『" $\theta \rrbracket \_i$ " es la incertidumbre asociada a realizar la acción para consumir el bien "n" del individuo "i" y la elasticidad de sustitución entre "n" y "y" es $1 /((1-\delta))$ 
Sujeto a la siguiente restricción presupuestaria:

$$
m=p_{i} n+y
$$

Donde $m$ es el ingreso, $p_{i}$ es el precio que el individuo "i" le asocia a "n", en términos de "y",

$p_{i}=\left\{\begin{array}{c}p, \text { si no ocurre un delito al realizar la acción para consumir "n" } \\ p(1+c), \text { si ocurre un delito al realizar la acción para consumir "n" }\end{array}\right.$

Donde "c" es el costo de haber sido víctima de un delito al realizar la acción para consumir "n", y la probabilidad subjetiva que el individuo "i" le asigna al desenlace de que ocurra un delito al realizar la acción para consumir "n" es "q".

Por tanto, utilizando la misma estrategia que Ayala y Chapa (2012) emplean, para resolver este problema de maximización, se sustituye la restricción presupuestaria en la función de utilidad y se calcula su esperanza matemática, lo cual resulta en la siguiente expresión:

$E(U)=q * \frac{[m-p(1-c) n]^{\delta}}{\delta}+(1-q) *\left[\frac{\theta n^{\delta}}{\delta}+\frac{(m-p n)^{\delta}}{\delta}\right]$

Posteriormente, se resuelven las condiciones de primer orden para obtener, la siguiente expresión:

$$
(1-q) \theta n^{\delta-1}=q p(1+c)[m-p n-c p n]^{\delta-1}+(1-q) p[m-p n]^{\delta-1}
$$

Y finalmente, resolviendo para " $n$ ", asumiendo que $\frac{c p n}{m-p n}=03$; se obtiene:

$n^{*}=\frac{\beta^{\sigma}}{\left(1+\beta^{\sigma} p\right)} * m$

Donde $\beta=\left[\frac{(1-q) \theta}{p(1+q c)}\right]^{\sigma}$ y $\sigma=\frac{1}{(1-\delta)}$

El resultado importante de este análisis teórico es que un incremento en $q$ provoca una disminución en $n^{*}$, lo cual quiere decir que la probabilidad subjetiva que las personas calculen para la ocurrencia de un delito, al realizar cierta acción para consumir algún bien, reducirá la demanda por ese bien.

\footnotetext{
${ }^{3}$ Ayala y Chapa (2012) consideran que este término no es más que un impuesto al valor que la delincuencia le impone al consumo del bien " $n$ " sobre el bien "y", equiparable con el impuesto al valor agregado, por lo que omitirlo no altera el propósito y las conclusiones del análisis teórico.
} 
La elección de las actividades económicas que podrían ser afectadas, por la percepción de la inseguridad y la tasa delictiva, está basada en la evidencia empírica que se describe a continuación.

El conocimiento de la violencia y el miedo excesivo pueden hacer que los habitantes de la zona geográfica afectada, migren hacia otros estados o municipios más seguros. La Encuesta de Percepción Ciudadana sobre Inseguridad en Ciudad Juárez sugieren que la percepción de la inseguridad podría ser una causa de migración. En 2009, el 42\% de la población de Ciudad Juárez manifestó, a través de la encuesta citada, su deseo de migrar para evitar ser víctimas de un delito. Adicionalmente, Robles et al. (2013), encuentra que un aumento de 10 homicidios por cada 100,000 habitantes genera una disminución aproximada de 2 a 3 puntos porcentuales de la proporción de personas que trabajan en un municipio. Por este motivo se considera que un incremento en la percepción de la inseguridad y la tasa delictiva podría provocar un incremento en la cantidad de personas que deciden emigrar fuera del país.

A su vez, Ayala y Chapa (2012) encuentran que las personas salen menos de su casa con fines recreativos, si perciben que la probabilidad de ser víctima de un delito en el acto, es alta. Tanto la percepción de la inseguridad como la tasa delictiva podrían desincentivar el consumo. Por este motivo, se considera que un incremento en la percepción de la inseguridad y la tasa delictiva podrían reducir el producto interno bruto de servicios de alojamiento temporal y de preparación de alimentos y bebidas en millones de MXN, y también afectarían al producto interno bruto de servicios de esparcimiento culturales y deportivos, $\mathrm{y}$ otros servicios recreativos en millones de MXN.

Estos factores pueden crear un ambiente poco propicio para los negocios. Una explicación viable es que en un área geográfica insegura el emprendedor teme por su propiedad y, por ende, disminuye la probabilidad de que decida abrir un negocio en esa zona, a menos que sea del giro de seguridad $u$ otro giro que no se vea afectado por estos factores. Además, es probable que los tres medios mencionados anteriormente harían menos atractiva casi cualquier tipo de inversión. La disminución de la productividad y la migración hacen más cara la mano de obra mientras que la austeridad reduce los retornos de la inversión. Robles et al. (2013), encuentra que un aumento de 10 homicidios por cada 100,000 habitantes genera que disminuya, en 0.4 puntos porcentuales, la proporción de personas propietarias de un negocio. Por este motivo, se considera un incremento en la percepción de la inseguridad y la tasa delictiva, que podrían reducir la inversión extranjera directa en millones de MXN y el número de empresas registradas en el Sistema de Información Empresarial Mexicano (SIEM). 


\section{Análisis empírico}

Para probar las diferencias de los efectos de la percepción de inseguridad y la tasa delictiva en la economía, se realizó un análisis de datos panel, tomando en consideración considerando los 32 estados de la república mexicana en el periodo 2011-2016. Esto resulta en un panel "balanceado" (i. e. que no le faltan observaciones) de 192 observaciones (32 objetos por 6 períodos de tiempo). Para controlar las variables que afectan a todos los estados y no cambian en el tiempo (e. g. ubicación, extensión territorial, etc.) y los "shocks" que afectan a todos los estados en cada período de tiempo, (e. g. una modificación de la tasa impositiva a nivel federal) se estimó el siguiente modelo estadístico de efectos fijos con una variable dicotómica, que identifica el año y otra que identifica el estado para cada una de los indicadores económicos que podrían ser afectados por la tasa delictiva o la percepción de la inseguridad. A continuación, se muestra dicho modelo estadístico, al que se denota Ecuación 7:

$\ln (\text { ind })_{i t}=\beta_{1} \ln (\text { per })_{i t}+\beta_{2} \ln (\text { inc })_{i t}+\alpha_{t}+\gamma_{i}+e_{i t}$

Donde "ind", se refiere a un indicador económico; "per", se refiere a percepción de la inseguridad tal y como se define en la introducción de este estudio; "inc", se refiere a la tasa de incidencia delictiva por cada 100 mil habitantes calculada por el INEGI con datos de la ENVIPE, " $\alpha$ " es una variable dicotómica para identificar el período, $\mathrm{t}=2011-2016$, " $\gamma_{i}$ " es una variable dicotómica para identificar el estado e " $i$ ", se refiere a cada uno de los 32 estados de la república mexicana. La "percepción de la inseguridad" es el porcentaje de encuestados que considera que vivir en el estado " $i$ " es inseguro.

Donde "ind", se refiere a un indicador económico; "per", se refiere a percepción de la inseguridad tal y como se define en la introducción de este estudio; "inc", se refiere a la tasa de incidencia delictiva por cada 100 mil habitantes calculada por el INEGI con datos de la ENVIPE, " $\alpha$ t" es una variable dicotómica para identificar el período, $t=2011-2016$, " $\gamma \_$”" es una variable dicotómica para identificar el estado e "i", se refiere a cada uno de los 32 estados de la república mexicana. La "percepción de la inseguridad" es el porcentaje de encuestados que considera que vivir en el estado " $\mathrm{i}$ " es inseguro.

Las estimaciones se hicieron a través de regresiones de mínimos cuadrados generalizados, utilizando el paquete estadístico R.

Cabe notar que este modelo no controla los impactos que afectan solo a unos estados (e. g. un arancel a la exportación de un producto que se produce en un estado); sin embargo, en el período de análisis, se considera que no hubo impactos de esta naturaleza que merezcan consideración. El Tratado de Libre Comercio de América del Norte no sufrió modificaciones en estos años, y no 
hubo desastres naturales que hayan causado suficientes estragos para ser tomados en cuenta.

Tabla 1

Signos esperados de los coeficientes de la percepción de la inseguridad y la tasa delictiva para cada uno de los indicadores económicos, considerados en los modelos estadísticos del presente estudio

\begin{tabular}{|c|c|c|c|}
\hline Indicador económico & Abreviación & Fuente & $\begin{array}{lrr}\text { Signo esperado } & \text { del } \\
\text { coeficiente de } & \text { la } \\
\text { percepción } & \text { de } & \text { la } \\
\text { inseguridad } & \text { y } & \text { la } \\
\text { tasa delictiva } & & \end{array}$ \\
\hline $\begin{array}{l}\text { Producto interno bruto de } \\
\text { servicios de alojamiento } \\
\text { temporal y de } \\
\text { preparación de alimentos } \\
\text { y bebidas en millones de } \\
\text { MXN }\end{array}$ & pibt & INEGI & $<0$ \\
\hline $\begin{array}{l}\text { Producto interno bruto de } \\
\text { servicios de } \\
\text { esparcimiento culturales } \\
\text { y deportivos, y otros } \\
\text { servicios recreativos en } \\
\text { millones de MXN }\end{array}$ & pibe & INEGI & $<0$ \\
\hline $\begin{array}{l}\text { Inversión Extranjera } \\
\text { Directa en millones de } \\
\text { MXN }\end{array}$ & ied & INEGI & $<0$ \\
\hline $\begin{array}{l}\text { Número de empresas } \\
\text { registradas en el Sistema } \\
\text { de Información } \\
\text { Empresarial Mexicano } \\
\text { (SIEM) }\end{array}$ & emp & SIEM & $<0$ \\
\hline $\begin{array}{l}\text { Personas que emigran } \\
\text { fuera de México }\end{array}$ & emi & CONAPO & $>0$ \\
\hline
\end{tabular}

Fuente: elaboración propia .

Idealmente, el modelo estadístico debería incluir los recursos naturales y las leyes del estado como variables explicativas, ya que ambas varían con el tiempo en cada estado. Ninguna de estas se incluye, porque ambas son difíciles de estimar, y se asume que en el período de análisis no variaron considerablemente.

A continuación, se presenta una tabla con el signo esperado del coeficiente, tanto de la percepción de la inseguridad como de la tasa delictiva que resulte al realizar las estimaciones del modelo estadístico, estimaciones correspondientes a cada uno de los indicadores económicos que podrían ser afectados por la percepción de la inseguridad y/o la tasa delictiva, junto con la definición del indicador económico, abreviación y fuente de este. El signo 
esperado de estos coeficientes se deriva de la relación esperada entre cada uno de los indicadores económicos con la percepción de la inseguridad y la tasa delictiva explicada, en la sección: “¿Cómo es que la percepción de la inseguridad y la tasa delictiva podrían afectar la economía?"

\section{Resultados}

En la siguiente tabla, se presentan los resultados de las estimaciones. Para la inversión extranjera directa ("ied"), el modelo estadístico que se utilizó fue el mismo que para el resto de los indicadores, tal y como se especifica en la Ecuación 7; pero sin logaritmos naturales, ya que este indicador puede tener valores negativos (lo cual significa una salida de capital) y, por ende, no se puede calcular su logaritmo natural.

Tabla 2

Resultados de la estimación de la Ecuación 7, para cada uno de los indicadores económicos considerados

\begin{tabular}{llll}
\hline ind & per & inc & R2 \\
\hline pibt & $-0.1127^{* *}$ & -0.0360 & 0.0383 \\
& $(0.0515)$ & $(0.0416)$ & \\
pibe & $-0.0233^{*}$ & -0.0091 & 0.0303 \\
& $(0.0124)$ & $(0.0101)$ & \\
ied & $-4.42 \mathrm{E}+02$ & $6.63 \mathrm{E}-03$ & 0.0075 \\
& $(6.16 \mathrm{E}+02)$ & $(7.54 \mathrm{E}-03)$ & \\
emp & $-0.5163^{* * *}$ & -0.1293 & 0.0926 \\
& $(0.1423)$ & $(0.1151)$ & 0.0339 \\
emi & $0.0155^{* *}$ & -0.0066 & \\
& $(0.0072)$ & $(0.0059)$ & \\
\hline
\end{tabular}

Nota: Error estándar en paréntesis. ${ }^{* * *}, * *, *$, se refiere a nivel de significancia estadística al $0.01,0.05$ y 0.1 , respectivamente. Para la inversión extranjera directa ("ied"), el modelo estadístico que se utilizó fue el mismo que para el resto de los indicadores, tal y como se especifica en la Ecuación 7; pero sin logaritmos naturales, ya que este indicador puede tener valores negativos (lo cual significa una salida de capital) y, por ende, no se puede calcular su logaritmo natural.

Tal y como se puede observar, solo los coeficientes de la percepción de la inseguridad son estadísticamente significativos, al menos, a un nivel de significancia estadística del 10\%. El único coeficiente de la percepción de la inseguridad que no es estadísticamente significativo, a un nivel de significancia estadística de al menos $10 \%$, es el correspondiente al modelo estadístico en el que la variable dependiente es la inversión extranjera directa en millones de MXN. Ninguno de los coeficientes de la tasa de incidencia delictiva por cada 100 mil habitantes, calculada por la INEGI con datos de la ENVIPE, es estadísticamente significativo a un nivel de significancia estadística de al menos 10\%. Todos los signos de los coeficientes estadísticamente significativos, al menos a un nivel de significancia estadística del 10\%, son los 
esperados. Un aumento del $10 \%$ en la percepción de la inseguridad reduce -en promedio- el producto interno bruto de servicios de alojamiento temporal y de preparación de alimentos y bebidas en millones de MXN, en $11.27 \%$. Un aumento del $10 \%$ en la percepción de la inseguridad reduce -en promedio- el producto interno bruto de servicios de esparcimiento culturales y deportivos, y otros servicios recreativos en millones de MXN, en $2.32 \%$. Un aumento del $10 \%$ en la percepción de la inseguridad reduce -en promedio- el número de empresas registradas en el SIEM, en 51.63\%. Un aumento del $10 \%$ en la percepción de la inseguridad incrementa -en promedio- la cantidad de personas que emigran fuera de México, en 1.55\%. Como es de notarse, el impacto de la percepción de la inseguridad es mayor en el número de empresas registradas en el SIEM y el producto interno bruto de servicios de alojamiento temporal y de preparación de alimentos y bebidas en millones de MXN.

\section{VI. ¿Y que hay de otras tasas delictivas?}

La tasa de incidencia delictiva por cada 100 mil habitantes, calculada por el INEGI con datos de la ENVIPE, refleja la cantidad de delitos que hay en cierta zona geográfica; sin embargo, puede ser que no todos los delitos afecten, en igual medida, el comportamiento de la población. Los delitos graves podrían afectar más el comportamiento de las personas que los delitos menos graves. Y de todos los delitos, el más grave para la sociedad considerando las penas asociadas al mismo, es el homicidio. ¿Qué pasaría si en lugar de utilizar tasa de incidencia delictiva por cada 100 mil habitantes calculada por el INEGI con datos de la ENVIPE, se utiliza la tasa de homicidios por cada $100 \mathrm{mil}$ habitantes calculada por el INEGI con datos de la ENVIPE, como variable independiente en los modelos estadísticos estimados en la sección anterior? Para ver qué sucedería, se procede a estimar la siguiente ecuación, denominada Ecuación 8:

$\ln (\text { ind })_{i t}=\beta_{1} \ln (\text { per })_{i t}+\beta_{2} \ln (\text { hom })_{i t}+\alpha_{t}+\gamma_{i}+e_{i t}$

Donde "ind" se refiere a un indicador económico, "per" se refiere a percepción de la inseguridad tal y como se define en la introducción de este estudio, "hom" se refiere a la tasa de homicidios por cada 100 mil habitantes calculada por el INEGI con datos de la ENVIPE, " $\alpha_{t}$ " es una variable dicotómica para identificar el período, $\mathrm{t}=2011-2016$, " $\gamma_{i}$ " es una variable dicotómica para identificar el estado e "i" se refiere a cada uno de los 32 estados de la república mexicana. La "percepción de la inseguridad" es el porcentaje de encuestados que considera que vivir en el estado " $i$ " es inseguro.

En la siguiente tabla, se presentan los resultados de las estimaciones: 
Tabla 3

Resultados considerando la tasa de homicidios por cada 100 mil

\begin{tabular}{llll}
\hline ind & per & hom & $\mathbf{R}^{\mathbf{2}}$ \\
\hline pibt & $-0.1116^{* *}$ & -0.0189 & 0.0392 \\
& $(0.0515)$ & $(0.0199)$ & \\
pibe & $-0.0217^{*}$ & $-0.0086^{*}$ & 0.0454 \\
& $(0.0124)$ & $(0.0048)$ & \\
ied & -310.6929 & -7.4884 & 0.0143 \\
& $(611.7620)$ & $(5.5437)$ & \\
emp & $-0.5507^{* *}$ & 0.0447 & 0.0890 \\
& $(0.1429)$ & $(0.0551)$ & \\
emi & 0.0116 & $0.0086^{* * *}$ & 0.0849 \\
& $(0.0071)$ & $(0.0027)$ & \\
\hline
\end{tabular}

Nota: Tasa de homicidios por cada 100 mil Habitantes calculada por la INEGI con datos de la ENVIPE (i. e. resultados de la estimación de la Ecuación 8 para cada uno de los indicadores económicos considerados). Error estándar en paréntesis. ${ }^{* * *},{ }^{* *},{ }^{*}$, se refiere a nivel de significancia estadística al $0.01,0.05$ y 0.1 , respectivamente. Para la inversión extranjera directa ("ied"), el modelo estadístico que se utilizó fue el mismo que para el resto de los indicadores, tal y como se especifica en la Ecuación 8, pero sin logaritmos naturales, ya que este indicador puede tener valores negativos (lo cual significa una salida de capital) y por ende, no se puede calcular su logaritmo natural.

Tal y como se puede observar, todos los signos son los esperados. En los modelos estadísticos que utilizan el producto interno bruto de servicios de alojamiento temporal y de preparación de alimentos y bebidas en millones de MXN y el número de empresas registradas en el SIEM como variables dependientes, respectivamente, solo la percepción de la inseguridad resulta tener un efecto estadísticamente significativo, al menos a un nivel de significancia estadística del $10 \%$. En el modelo estadístico que utiliza el producto interno bruto de servicios de esparcimiento culturales y deportivos, y otros servicios recreativos en millones de MXN como variable dependiente, la percepción de la inseguridad y la tasa de homicidios por cada 100 mil habitantes tienen efectos estadísticamente significativos con el mismo nivel de significancia estadística. Sin embargo, en este caso, la percepción de la inseguridad afecta más al producto interno bruto de servicios de esparcimiento culturales y deportivos, y otros servicios recreativos en millones de MXN que la tasa de homicidios por cada 100 mil habitantes. Un aumento del $10 \%$ en la percepción de la inseguridad reduce, en promedio, el producto interno bruto de servicios de esparcimiento culturales y deportivos y otros servicios recreativos en millones de MXN, en $2.17 \%$ mientras que un aumento del $10 \%$ en la tasa de homicidios solo lo reduce $0.86 \%$, en promedio. Únicamente en el modelo estadístico que utiliza las personas que emigran fuera de México, como variable dependiente, la percepción de la inseguridad no tiene un efecto estadísticamente significativo, y la tasa de homicidios por cada 100 mil habitantes, sí. Sin embargo, el efecto de la tasa de homicidios es pequeño. Un aumento del $10 \%$ en la tasa de homicidios incrementa la cantidad de personas 
que emigran fuera de México $0.86 \%$, en promedio. El único modelo estadístico que no presenta coeficientes estadísticamente significativos es el que utiliza la inversión extranjera directa, como variable dependiente.

La tasa de incidencia delictiva por cada 100 mil habitantes calculada por el INEGI con datos de la ENVIPE refleja la cantidad de delitos que hay en cierta zona geográfica; sin embargo, puede ser que los delitos estén concentrados en ciertas partes de la zona geográfica $\mathrm{y}$, por ese motivo, no afectan la actividad económica en toda la zona. Esta es una posible explicación de por qué los coeficientes relacionados con la tasa de incidencia delictiva por cada 100 mil habitantes, calculada por el INEGI con datos de la ENVIPE, no resultaron ser estadísticamente significativos a un nivel de significancia estadística de al menos $10 \%$, en las estimaciones realizadas con la ecuación 7 . La tasa de prevalencia delictiva por cada 100 mil habitantes refleja cuántas personas son afectadas por al menos un delito, en una zona geográfica. ¿Qué pasaría si, en lugar de utilizar la tasa de incidencia delictiva por cada 100 mil habitantes calculada por el INEGI con datos de la ENVIPE, se utiliza la tasa de prevalencia delictiva por cada 100 mil habitantes calculada por el INEGI con datos de la ENVIPE, como variable independiente en los modelos estadísticos estimados en las secciones anteriores? Para ver qué sucedería, se procede a estimar la siguiente ecuación denominada Ecuación 9:

$\ln (\text { ind })_{i t}=\beta_{1} \ln (\text { per })_{i t}+\beta_{2} \ln (\text { prev })_{i t}+\alpha_{t}+\gamma_{i}+e_{i t}$

Donde "ind", se refiere a un indicador económico; "per", se refiere a percepción de la inseguridad tal y como se define en la introducción; "prev", se refiere a la tasa de prevalencia delictiva por cada 100 mil habitantes calculada por el INEGI con datos de la ENVIPE; " $\alpha_{t}$ " es una variable dicotómica para identificar el período; $\mathrm{t}=2011-2016$; " $\gamma_{i}$ " es una variable dicotómica para identificar el estado e "i", se refiere a cada uno de los 32 estados de la república mexicana. La "percepción de la inseguridad" es el porcentaje de encuestados que considera que vivir en el estado "i", es inseguro.

En la siguiente tabla se presentan los resultados de las estimaciones. Para la inversión extranjera directa ("ied"), el modelo estadístico que se utilizó fue el mismo que pare el resto de los indicadores tal y como se especifica en la Ecuación 9 pero sin logaritmos naturales ya que este indicador puede tener valores negativos (lo cual significa una salida de capital) y por ende no se puede calcular su logaritmo natural. 
Tabla 4

Resultados considerando la tasa de prevalencia delictiva por cada 100 mil habitantes

\begin{tabular}{cccc}
\hline ind & per & prev & $\mathbf{R}^{2}$ \\
\hline pibt & $-0.1133^{* *}$ & -0.0227 & 0.0343 \\
& $(0.0529)$ & $(0.0653)$ & \\
pibe & -0.0209 & -0.0178 & 0.0333 \\
& $(0.0128)$ & $(0.0157)$ & \\
ied & $-4.90 \mathrm{E}+02$ & $1.09 \mathrm{E}-02$ & 0.0052 \\
& $(636.3010)$ & $(0.0170)$ & 0.0924 \\
emp & $-0.4939 * * *$ & -0.2006 & 0.0286 \\
& $(0.1462)$ & $(0.1804)$ & \\
\hline \multirow{2}{*}{ emi } & $0.0158 * *$ & -0.0062 & $(0.0092)$ \\
& $(0.0075)$ &
\end{tabular}

Nota: Tasa de prevalencia delictiva por cada 100 mil habitantes calculada por la INEGI con datos de la ENVIPE (i. e. resultados de la estimación de la Ecuación 9 para cada uno de los indicadores económicos considerados). Error estándar en paréntesis. $* * *, * *,{ }^{*}$, se refiere a nivel de significancia estadística al $0.01,0.05$ y 0.1 respectivamente.

Tal y como se puede observar, solo los coeficientes de la percepción de la inseguridad son estadísticamente significativos al menos a un nivel de significancia estadística del 5\%. Los únicos coeficientes que no son estadísticamente significativos al menos a un nivel de significancia estadística del $10 \%$ son los correspondientes a los modelos que tienen como variable dependiente el producto interno bruto de servicios de esparcimiento culturales $\mathrm{y}$ deportivos, y otros servicios recreativos en millones de MXN y la inversión extranjera directa en millones de MXN respectivamente. Ninguno de los coeficientes de la tasa de prevalencia delictiva por cada 100 mil habitantes calculada por la INEGI con datos de la ENVIPE es estadísticamente significativo a un nivel de significancia estadística de al menos $10 \%$. Todos los signos de los coeficientes estadísticamente significativos al menos a un nivel de significancia estadística del 5\% son los esperados. Un aumento del $10 \%$ en la percepción de la inseguridad disminuye en promedio el producto interno bruto de servicios de alojamiento temporal y de preparación de alimentos y bebidas en millones de MXN en $11.33 \%$. Un aumento del $10 \%$ en la percepción de la inseguridad disminuye en promedio el número de empresas registradas en el SIEM 49.39\%. Un aumento del 10\%, en la percepción de la inseguridad, aumenta en promedio la cantidad de personas que emigran fuera de México $1.57 \%$. Como es de notarse, el impacto de la percepción de la inseguridad es mayor en el número de empresas registradas en el SIEM, y el producto interno bruto de servicios de alojamiento temporal y de preparación de alimentos y bebidas, en millones de MXN. 


\section{Conclusión}

Es difícil imaginar que una persona vaya a revisar las tasas delictivas reportadas por el Ministerio Público para tomar la decisión de ir a pasear al parque, por ejemplo. Además, las tasas delictivas representan una información difícil de incluir en la toma de este tipo de decisiones. Si la persona es suficientemente sofisticada, entenderá que este estadístico está sesgado por la "cifra negra". Es decir, por todos los delitos que no se reportan por vergüenza, pereza, corrupción o falta de confianza en las autoridades. Para ello, tendrá que recurrir al resultado de encuestas como la ENVIPE y considerar todos los sesgos inherentes en estos estudios (e. g., la memoria y el estado de ánimo del encuestado a la hora de contestar la encuesta, su disponibilidad, cómo la redacción de la pregunta podría inclinar al encuestado a contestarla de cierta forma, etc.). Esto, además de ser más costoso, es muy demandante para la mente, por ende, propenso a errores de cálculo o limitantes cognitivas.

Es más fácil imaginar que la persona utilice una heurística para contestar la difícil pregunta de: ¿qué tan probable es que me roben la billetera si salgo a dar un paseo al parque? Dicha estrategia podría sustituir esta pregunta por otras más sencillas como: ¿salen a pasear al parque mis vecinos? Si veo que lo hacen, no debe haber problema con esta actividad y, por ende, la probabilidad de que me roben la billetera en el acto debe ser mínima. Otra pregunta que se podría utilizar en sustitución, es: ¿en qué condiciones está el parque?, si noto que está afectado por actos vandálicos, es probable que no haya patrullaje por allí, por tanto, la probabilidad de que me roben la billetera es alta. De hecho, Lewis y Maxfield (1980) encuentran que en Chicago la cantidad de edificios abandonados y el vandalismo juvenil afectan más la percepción de la inseguridad que las tasas delictivas.

Considerando las limitantes estadísticas de este análisis (las variables que no se pudieron incluir en los modelos estadísticos y el resultado tan bajo de sus respectivos coeficientes de determinación), se puede concluir que la percepción de la inseguridad afecta más la economía que las tasas delictivas consideradas en este estudio. Esto implica que, si los gobiernos quieren ver sus esfuerzos para combatir la inseguridad reflejados en la economía, tendrán que considerar reducir la percepción de la inseguridad, sin olvidar reducir las tasas delictivas y poner más énfasis en lo primero que en lo segundo. Cualquiera pensaría que patrullar los parques recreativos es una buena política para disminuir tanto la tasa delictiva como la percepción de la inseguridad, sin embargo, tal y como encuentra López (2013), este no es el caso. Contrario a las expectativas, mucha gente no se da cuenta de esta medida y la percepción de la inseguridad se mantiene. 
Todas las estimaciones realizadas en este estudio indican que la percepción de la inseguridad afecta más al número de empresas registradas en el SIEM y al producto interno bruto de servicios de alojamiento temporal y de preparación de alimentos y bebidas en millones de MXN. Un incremento del $10 \%$ en la percepción de la inseguridad está relacionado con una disminución -en promedio- de aproximadamente el 50\% del número de empresas registradas en el SIEM, y una disminución -en promedio- de aproximadamente $11 \%$ del producto interno bruto de servicios de alojamiento temporal y de preparación de alimentos y bebidas en millones de MXN.

Aunado a esto, tanto la tasa delictiva como la percepción de la inseguridad podrían afectar la economía de otras maneras. Por ejemplo, Pope (2008) encuentra que el precio de las casas en ciertos barrios de Estados Unidos, baja cuando un delincuente sexual se muda al vecindario y aumentan cuando sale del mismo. Para futuras investigaciones habrá que realizar el análisis para otros indicadores económicos según sugiera la evidencia empírica.

A pesar de que los coeficientes de correlación distancia, entre la matriz de las percepciones de inseguridad contra la matriz de las tasas de incidencia delictiva por cada 100 mil habitantes, calculadas por el INEGI con datos de la ENVIPE, la matriz de las tasas de homicidios por cada 100 mil habitantes calculadas por el INEGI con datos de la ENVIPE y la matriz de las tasas de prevalencia delictiva por cada 100 mil habitantes calculadas por el INEGI con datos de la ENVIPE, respectivamente, no pasan de 0.21 , sin embargo, puede ser que la tasa delictiva afecte la actividad económica a través de la percepción de la inseguridad. Para futuras investigaciones, será interesante realizar un análisis de mediación con datos panel para probar esta hipótesis. El principal reto de este análisis de acuerdo con Little (2013) será determinar cuánto tiempo tarda la tasa delictiva en afectar la percepción de la inseguridad y cuánto tiempo tarda la percepción de la inseguridad en afectar la actividad económica si es que realmente la tasa delictiva afecta la actividad económica a través de la percepción de la inseguridad. Además, habrá que revisar si no hay otras variables mediadoras involucradas en la cadena de causalidad. Puede ser que la tasa delictiva afecte el miedo a ser víctima de un delito, y que el miedo a ser víctima de un delito afecte la percepción de la inseguridad y que, a su vez, la percepción de la inseguridad afecte la actividad económica.

\section{Referencias}

[1] Ayala Gaytán, E. A., \& Chapa Cantú, J. C. (2012). La inseguridad y la demanda por entretenimiento: evidencia para las áreas metropolitanas de Monterrey, Guadalajara y León, EconoQuantum, 9(1), 135-148

[2] Bazerman, M. H., \& Moore, D. A. (2008). Judgment in managerial decision making. 
[3] Curiel, R. P., \& Bishop, S. R. (2016). A metric of the difference between perception of security and victimisation rates. Crime Science, 5(1), 12.

[4] ENOPI (Junio 2017). Encuesta Nacional de Opinión Pública Ipsos. Recuperado de: https://www.ipsos.com/

[5] Gilovich, T., Griffin, D., \& Kahneman, D. (Eds.). (2002) Heuristics and biases: The psychology of intuitive judgment. Cambridge university press.

[6] Hale, C. (1996) Fear of Crime: A Review of the Literature, International Review of Victimology, 4: 79-150.

[7] Lewis, D. A., \& Maxfield, M. G. (1980). Fear in the neighborhoods: An investigation of the impact of crime. Journal of research in crime and delinquency, 17(2), 160-189.

[8] Little, T. D. (2013). Longitudinal structural equation modeling. Guilford press.

[9] López, C. J. (2013). Percepción de inseguridad en México. Revista Mexicana de Opinión Pública, 15, 12-29.

[10] Pope, J. C. (2008). Fear of crime and housing prices: Household reactions to sex offender registries. Journal of Urban Economics, 64(3), 601-614.

[11] Robles, G., Calderón, G., \& Magaloni, B. (2013). Las consecuencias económicas de la violencia del narcotráfico en México (No. IDB-WP-426). IDB Working Paper Series

[12] Szekely, G. J., Rizzo, M. L. \& Bakirov, N. K. (2007). Measuring and testing dependence by Correlation of distances. The Annals of Statistics, 35(6): 27692794.

[13] Tversky, A., \& Kahneman, D. (1973). Availability: A heuristic for judging frequency and probability. Cognitive psychology, 5(2), 207-232. 
